\title{
Self-Assembly of Cellulose Nanocrystals and Organic Colored Pigments as Reinforcement Matrix of Lipstick for Enhancing SPF
}

\author{
Lidan Xiong, ${ }^{1,2}$ Hailun He $\mathbb{D}^{1,3}$ Jie Tang, ${ }^{1,2}$ Qi Yang, ${ }^{4}$ and Li Li $\mathbb{D}^{1,2,3}$ \\ ${ }^{1}$ Cosmetics Safety and Efficacy Evaluation Centre, Sichuan University West China Hospital, 610041, China \\ ${ }^{2}$ Sichuan Engineering Technology Research Centre of Cosmetic, 610041, China \\ ${ }^{3}$ Department of Dermatology, Sichuan University West China Hospital, 610041, China \\ ${ }^{4}$ Department of Polymer Science and Engineering, Sichuan University, 610041, China \\ Correspondence should be addressed to Li Li; lilihx_scu@scu.edu.cn
}

Received 15 July 2021; Revised 13 January 2022; Accepted 17 January 2022; Published 9 February 2022

Academic Editor: Md Saquib Hasnain

Copyright (c) 2022 Lidan Xiong et al. This is an open access article distributed under the Creative Commons Attribution License, which permits unrestricted use, distribution, and reproduction in any medium, provided the original work is properly cited.

\begin{abstract}
The vermilion of the human lip, covered by a skinny epithelium with little melanin, is quite susceptible to damage from ultraviolet (UV) radiation exposure. However, commercial sunscreen filters and indelible dyes used in lipsticks can cause health hazards after percutaneous absorption or accidentally oral administration. Inspired by plant pigmentation as natural filters to protect themselves against overexposure to UV, safer bio-based sunscreens of cellulose enveloped with anthocyanin (AN) were developed using bionic design. Cellulose nanocrystals (CNC), derived from acid hydrolysis of cellulose, reinforced enhancement of UV absorption and shielding properties of AN. This innovation addresses the issue that naturally sourced UV filter application to sunscreen does not achieve a desired sun protection factor (SPF) value because of the low specific extinction value $(\mathrm{E} 1,1)$. We also stated that the diverse formula of anthocyanin sunscreen lipsticks with $\mathrm{CNC}$ exhibited 10 times more SPF value than AN alone. Furthermore, they possess competitive benefits such as pleasing texture, superior adhesion, impermeable, nonphototoxicity, ease of application, and removal. This work provides a promising proof-of-concept for studying the features of natural sunscreens in the design of simple, safe, efficient, and green sunscreens.
\end{abstract}

\section{Introduction}

Chronic exposure to ultraviolet (UV) radiation is known to damage the structure and function of the skin. It has long been recognized that using artificially synthesized sunscreens to protect the skin as long ago as the 19th century [1]. As recently as 1985, the significance of UV-induced lip cancer was recognized $[2,3]$. The lips are covered by a very thin orthokeratotic horny layer, containing little melanin. Thus, they are highly sensitive to chemical and physical insults, especially UV-exposed lip skin. In particular, UV radiation damages human lips, including photoaging, actinic cheilitis, and malignant tumor $[4,5]$. In comparison, oral exposure to UV radiation is associated with a higher risk of cancer than skin exposure [6].

Dating back to ancient Egypt and the Tang Dynasty of China, traditional rouge, roughly equivalent to modern lip- stick, was formulated from nontransparent minerals and plants such as clove and cinnabar (Figure 1(a)). Currently, the sunscreen filters in lipstick consist primarily of artificial chemicals, such as oxybenzone, avobenzone, 4methylbenzylidene camphor, octocrylene, and inorganic filters, such as titanium dioxide and zinc oxide [7]. However, there are some controversies and side effects associated with these agents, which can penetrate through the epidermis or even be absorbed into the bloodstream leading adverse health effects on humans [8-12]. Specifically, the risk of synthetic UV actives included in lipstick is increased when they are accidentally taken orally. In general, natural sunscreens are likely to exhibit fewer detrimental side effects.

During the path of evolution, plants have evolved efficacious safeguard mechanisms against overexposure to solar radiation. Flavonoids, especially anthocyanin (AN), are the main anti-UVB polysaccharides that have demonstrated 


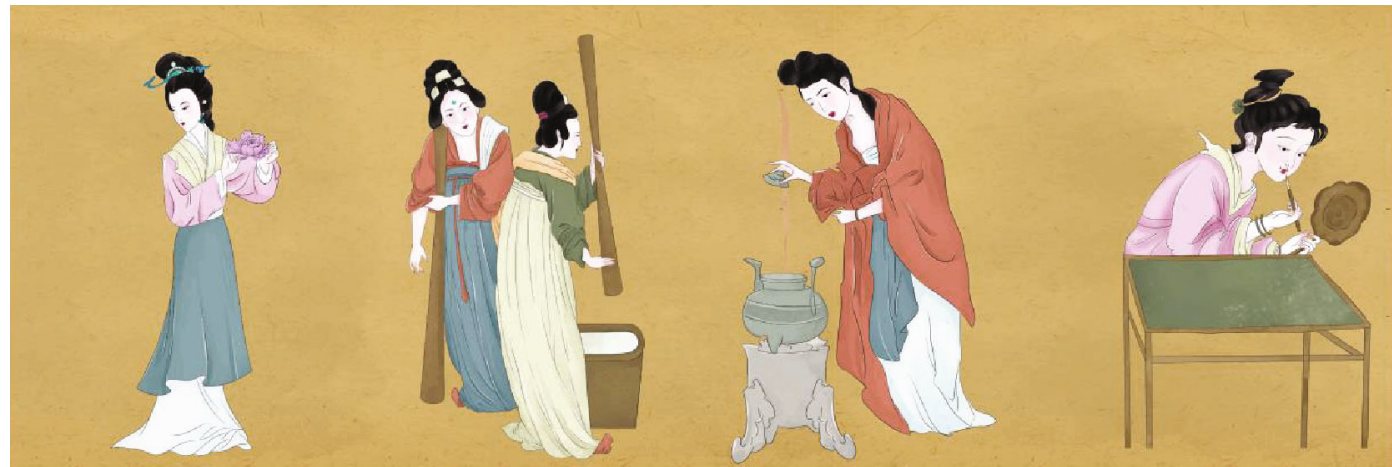

(a)
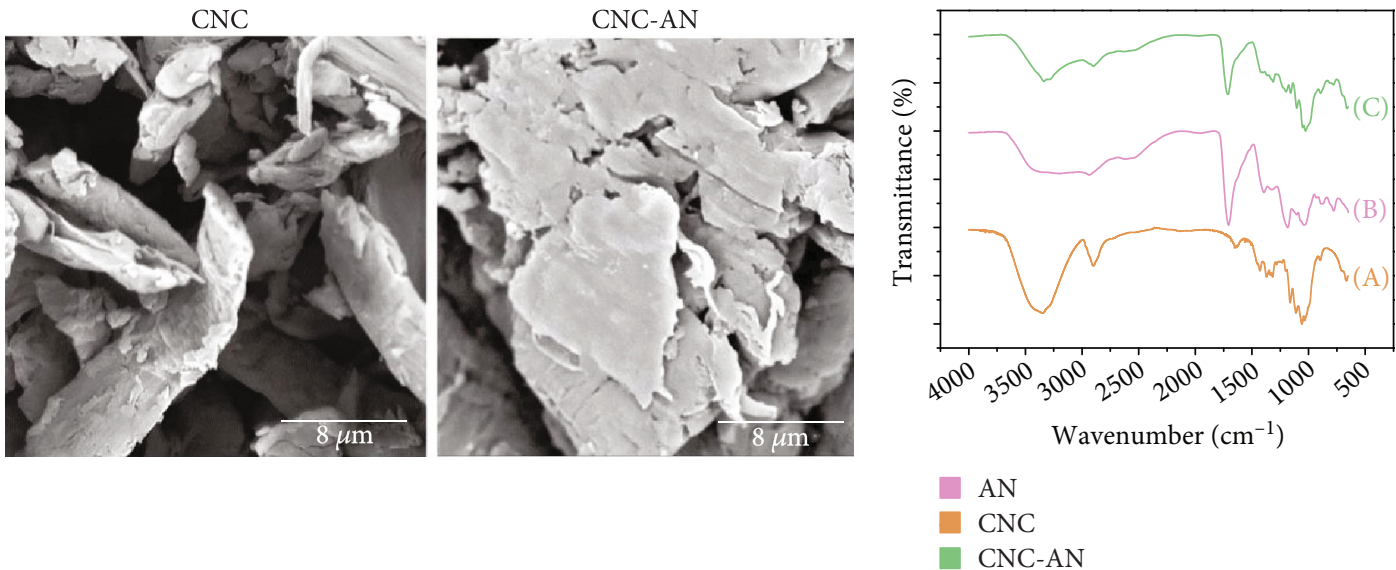

(b)
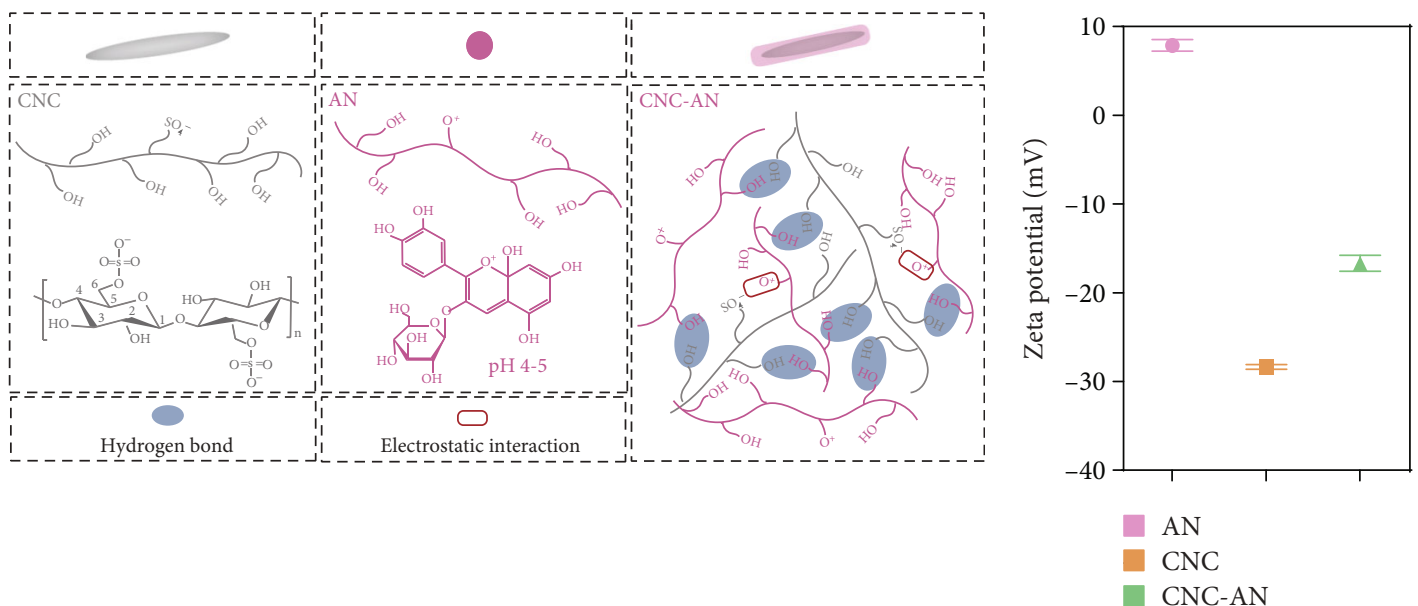

(d)

(e)

FIgURE 1: (a) The lipstick made by traditional ancient methods. (b) Scanning electron microscopic (SEM) images of CNC and CNC-AN. (c) The Fourier transform infrared spectrometer (FTIR) was applied to evaluate the CNC, AN, and CNC-AN. (d) The synthetic scheme toward $\mathrm{CNC}$ and $\mathrm{AN}$ via electrostatic adsorption and hydrogen bond. (e) Zeta potential of CNC, AN, and CNC-AN.

chemopreventive effect via $\pi$-stacking interactions [13]. Moreover, anthocyanin shows color variations under different $\mathrm{pH}$ values [14], which can create color reviver balm because of different $\mathrm{pH}$ values in human lips [15]. Previous studies have documented that many polyphenols with aromatic moieties possess anti-UV radiation activity, but the inherent variation in bioactivity, concentration, and specific extinction value $(\mathrm{E} 1,1)$ poses some shortcomings on the application in sunscreen products [15]. Currently, none of the reported botany sunscreen exhibit sun protection factor (SPF) values higher than 30 [16]. To overcome this challenge, it is therefore urgent to develop a safe and effective sunscreen lipstick so as to avoid hazardous chemical filters and dyestuff constituents.

In this work, our effort was focused on design and preparation of CNC-AN lipstick sunscreens that were safe and 
simple for human lip photoprotection. Inspired by natural fibers of textile products, cellulose from hemp stalk enveloped with AN UV absorbers in the vacuoles was developed using bionic design [17]. Binding of anthocyanins to acid hydrolysis of cellulose, the lipstick exhibited pleasing texture due to the reddish color. Remarkably, mimicking native plant cell wall binding with AN could lead to a significant increase of UV-adsorption, UV-shielding, and SPF value, probably because UV-filters binding with high surface area cellulose changed the physical properties of organic particulate UV absorbers such as size or light path [18]. Further, the sunblock lipstick based on AN-CNC contained none commercial UV-filters. The novel biopolymer sunscreen lipsticks, avoiding ingesting synthetic sunscreen and dyestuff constituents, were fulfilling food-grade, impermeable, and nonphototoxicity.

\section{Material and Methods}

2.1. Materials. Red cabbage (Brassica oleracea L. var capitata f. rubra) was purchased from a local supermarket, while Pectinase and Cyanidol-3-Glucoside were obtained from Aladdin (Shanghai, China). Hemp stalk was provided as a present. In addition, cationic hydroxyethyl cellulose (cHEC), CCK-8 (Cell Counting Kit-8), fluorescein isothiocyanate (FITC), and $2^{\prime}, 7^{\prime}$-dichlorohydrofluorescein diacetate (DCFH-DA) were acquired from Usolf (Qingdao, China), Dojindo (Dojindo Laboratories, Japan), and Sigma Chemical Co. (Sigma, USA), respectively. Anti- $\gamma \mathrm{H} 2 \mathrm{AX}$ was obtained from Abcam (Abcam, UK). DMEM, penicillin, and streptomycin solution were purchased from HyClone (GE Health Care Life Science, USA), while B27 was obtained from Gibco (Thermo Fisher, USA). BALB/c mice and nude mice were purchased from Dashuo Biological Technology Co., Ltd. (Chendu, China).

\subsection{Methods}

\subsubsection{Preparation and Characterization of CNC-AN}

(1) Preparation of CNC-AN Homogenate and Freeze-Dried Powders. CNC extracted using acid hydrolysis of hemp stem was added into anthocyanin liquid at $0.15 \mathrm{~g} / \mathrm{mL}$ and then stirred at $60^{\circ} \mathrm{C}$ for $30 \mathrm{~min}$. Then, the cellulose was centrifuged at $2000 \mathrm{rpm}$ for $15 \mathrm{~min}$ [19]. The precipitation was grafting CNC-AN homogenate and eventually freeze-dried.

(2) Morphological and Fourier Transform Infrared (FTIR) Examination. CNC and CNC-AN that were taken were subjected to morphological study using scanning electron microscopy (SEM) Phenom Pro (phenom world, Netherlands) at an acceleration voltage of $10 \mathrm{kV}$. Then, we compared CNC-AN with CNC and AN using FTIR (FT-IR, Nicolet 6700, Thermo Fisher Scientific, USA).

(3) UV Absorption and Shielding Determination. All band ultraviolet scanning for measuring UV absorption was determined using a spectrophotometer (Thermo Fisher, USA). We detected UV shielding rate using UV-LED light (Sigma,
USA) and UV irradiance meter (National Institute of Metrology, China).

(4) Zeta Potentials and Bioadhesion Determination. Zeta potentials of $\mathrm{CNC}, \mathrm{AN}$, and $\mathrm{CNC}-\mathrm{AN}$ were detected using Zeta View (Particle Metrix, Germany). To observe the bioadhesion, $\mathrm{CNC}, \mathrm{AN}$, and $\mathrm{CNC}-\mathrm{AN}$ were smeared on the inner forearm and took pictures for $15 \mathrm{sec}$ continuously.

2.2.2. Fabrication and Characterization of CNC-AN Lip Gloss and Lipstick. For this procedure, we briefly added $0.5 \mathrm{~g} \mathrm{CNC}-\mathrm{AN}$ freeze-dried powder into $6.6 \mathrm{~g}$ soft oil. After evenly dispersed, we added $2.1 \mathrm{~g}$ hard oil and stirred it at $120^{\circ} \mathrm{C}$. When the hard oil was completely melted, we then poured it into the mold to formulate lipstick [20]. Additionally, CNC-AN, AN, and cHEC were mixed at ratios of $10: 10: 1$, stirred evenly at $60^{\circ} \mathrm{C}$, and then cooled down to room temperature to obtain lip gloss. The stability of lipstick and lip gloss was studied at $40^{\circ} \mathrm{C}$ and $-15^{\circ} \mathrm{C}$. The analyses were conducted placing samples of lipstick and lip gloss (final pH 6.5-6.8) in an electrothermal incubator or refrigerator for $24 \mathrm{~h}$. In order to explore the stability of formulations for 3 months, lipstick and lip gloss were kept in stability cabinet (Shang Hai Jinhong, China) $50 \pm 0.1^{\circ} \mathrm{C}$ and $60 \%$ relative humidity and in refrigerator $-5 \pm 0.1^{\circ} \mathrm{C}$. Finally, we captured SEM images of CNC-AN, lip gloss, and lipstick using Phenom Pro (phenom world, Netherlands) at an acceleration voltage of $10 \mathrm{kV}$.

\subsubsection{In Vitro Evaluation}

(1) SPF Determination. The SPF value of the lipstick and lip gloss was assessed by SPF 290S (Optometric, USA) [21]. The sample was sprinkled on the $3 \mathrm{M}$ film with a needle syringe $\left(2 \mathrm{mg} / \mathrm{cm}^{2}\right.$ sample layer). Then, it was placed at room temperature for 30 minutes in the dark for SPF determination.

(2) Cell Culture. Here, the human keratinocyte cell line (HaCaT) was acquired from China Center for Type Culture Collection (Kunming, China). HaCaT was cultured in RPMI DMEM media (Dulbecco's Minimum Essential Medium), supplemented with $100 \mathrm{U} / \mathrm{mL}$ penicillin, $100 \mu \mathrm{g} / \mathrm{mL}$ streptomycin, and 10\% FBS (Gibco). Then, the cells were incubated at $37^{\circ} \mathrm{C}$ in $5 \% \mathrm{CO}_{2}$ [22].

(3) Cell Viability Assay. HaCaT $\left(3.0 \times 10^{4}\right.$ cells/well $)$ were seeded into 96 -well plates. Cultures were preprocessing with varying concentrations of anthocyanin for $24 \mathrm{~h}$. Subsequently, we added CCK-8 solution to each well for $3 \mathrm{~h}$. Absorbance was measured at $450 \mathrm{~nm}$ using a microplate spectrophotometer (Bio-Rad Inc., USA) [22].

(4) Protection of UVB-Induced Oxidation Impact In Vitro. The intracellular reactive oxygen species (ROS) level, which can be measured with the fluorescence intensity of DCFHDA, was applied to evaluate antioxidant ability [22]. HaCaT was incubated in the 6-well plate and was assigned to control, UVB, sunscreen, lipstick, and lip gloss groups, respectively. The sunscreen, lipstick, and lip gloss were applied 
on a slide that can penetrate UVB, whereas $\mathrm{HaCaT}$ was exposed to $60 \mathrm{~mJ} / \mathrm{cm}^{2}$ UVB leading to $60 \%$ cell viability. Then, cells were incubated with DCFH-DA for $60 \mathrm{~min}$ at $37^{\circ} \mathrm{C}$. We finally recorded the fluorescence images using a fluorescent inverted microscope (Olympus IX71, Japan).

(5) Protection of UVB-Induced DNA Damage In Vitro. The DNA break in HaCaT cells can be detected using a comet assay [22]. First, place the first layer of the regular melting agar sugar (LMA) in the oven at $60^{\circ} \mathrm{C}$. Then, $10 \mu \mathrm{L}$ cells and $75 \mu \mathrm{L} 0.7 \%$ low melting agar sugar (LMA) were the second layer of LMA at $4^{\circ} \mathrm{C}$ for $10 \mathrm{~min}$ to solidify. After lysis, electrophoresis, neutralization, and staining, the images were recorded using fluorescence microscopy. The percentage of tail DNA\% was evaluated with CASP1.2.3 (Casplab, Poland). To evaluate the protection of UVB-induced nucleus damage by CNC-AN lipstick and lip gloss, $\gamma \mathrm{H}_{2} \mathrm{AX}$ cell immunofluorescence was applied [22]. HaCaT cells in 24-well plates were classified into control, UVB, sunscreen, lipstick, and lip gloss groups. Every group occupied 3 wells. After paraformaldehyde fixation, $\gamma \mathrm{H}_{2} \mathrm{AX}$ antibody, and secondary antibody incubation, the fluorescence images were recorded under a fluorescent inverted microscope (Olympus IX71, Japan).

(6) Organotypic Cultures. BALB/c mice were sacrificed using $\mathrm{CO}_{2}$ asphyxiation, and the lip was harvested and sterilized with $70 \%$ alcohol. We placed the lip clippings into cold PBS solution and cut the excess skin section off. Then, lip explants were incubated epidermal-side-up on a transwell in 6-well plates. The cell culture media consisted of DMEM (low glucose), supplemented with B-27, 25 units/mL penicillin, and $25 \mathrm{mg} / \mathrm{mL}$ streptomycin. Organotypic cultures were maintained in a $5 \% \mathrm{CO}_{2}$ incubator at the temperature of $37^{\circ} \mathrm{C}$ for $24 \mathrm{~h}$. Five wells were allocated to control, UVB, sunscreen, lipstick, and lip gloss groups, respectively. Then, lips were exposed to $5040 \mathrm{~mJ} / \mathrm{cm}^{2}$ UVB. After irradiation, the lips were incubated in a $5 \% \mathrm{CO}_{2}$ incubator at the temperature of $37^{\circ} \mathrm{C}$ for $72 \mathrm{~h}$. The lips of mice were collected to stain hematoxylin and eosin (HE) and taken images by a microscope.

(7) Transdermal Penetration in Pigskin. FITC grafting freeze-dried powder was applied to prepare lipstick and lip gloss. $100 \mu \mathrm{m}$ thick lipstick and lip gloss were applied to the pigskin, while PBS with free FITC was used as positive control. After $6 \mathrm{~h}$, the samples were frozen in a frozen section compound (FSC 22, Leica Microsystems, Buffalo Grove, USA) and vertically cut into $4 \mu \mathrm{m}$ thick slices using Cryostat microtome (Leica, Mainz, Germany) at $-20^{\circ} \mathrm{C}$ [23]. Then, the sections were imaged under a microscope (Olympus, Tokyo, Japan), while the depth of fluorescence penetration was measured using ImageJ 7.0.

\subsubsection{Animal Experiments}

(1) Evaluation of Lipstick and Lip Gloss Skin Adhesion and Easy Wiping. 0.1 g CNC-AN freeze-dried powder was dis- solved in $3 \mathrm{~mL}$ DMSO, followed by the addition of $0.6 \mathrm{~mL}$ FITC/DMSO $(0.01 \mathrm{~g} / \mathrm{mL})$ and $0.2 \mathrm{~g}$ Ditin butyl dilaurate (DBTDL). After a water bath at $100^{\circ} \mathrm{C}$ for $4 \mathrm{~h}$, the reaction mixture was precipitated, dried, dialyzed, and frozen. Afterward, three nude mice were used to evaluate the adhesion of lipstick and lip gloss. FITC grafting freeze-dried powder was used to prepare lipstick and lip gloss (commercial lipstick as a positive control) [24]. Using IVIS Spectrum (Caliper Life Sciences) [25], we imaged and quantified lipstick, lip gloss, and commercial lipstick remaining on the skin at $0,2,4$, and $6 \mathrm{~h}$, before and after being washed with water or wiped a wet towel.

(2) UV Radiation and Lipstick Treatment. The animal experiments were approved by the ethics committee of West China Hospital following the National Institutes of Health guidelines for the care and use of laboratory animals. BALB/c mice were divided into three groups $(n=2$ each), where 1 was for lipstick, while the other was for lip gloss. Then, the back of mice was divided into 4 sections (control, UVB, sunscreen with UVB, lipstick with UVB, and lip gloss with UVB, at $1 \mathrm{~cm} \times 1 \mathrm{~cm} \times 100 \mu \mathrm{m}$ film). Then, the mice were exposed to $5000 \mathrm{~mJ} / \mathrm{cm}^{2}$ UVB. After irradiation, the mice of the 3 groups were observed for another 24,48 , and $72 \mathrm{~h}$. In this subsection, the back skin and lips of mice were collected. After fixation, embedding, dewaxing, hydration, and rinse, the sections were immersed in hematoxylin and eosin staining solution. Morphology was photographed under a microscope.

\subsubsection{Human Evaluation}

(1) Preparation for Human Evaluation. Twenty people aged 20-45 years from Chengdu were enrolled in this study. The human experiments were approved by the ethics committee of West China Hospital according to the National Institutes of Health guidelines for care.

(2) CIE 3D Space System. We used the CIE 3D space system to evaluate the adhesion of lipstick and lip gloss [26]. The lipstick, lip gloss, and commercial lipstick were applied to the skin. The values of $L *$ and $a *$ were detected at $0,1,2$, $3,4,5,6,7$, and $8 \mathrm{~h}$.

(3) Dermoscopy. To discover whether the lipstick and lip gloss can be removed with water or a wet towel, the CBS606 portable dermatoscope (Xiangmei Technology, Taiwan, China) was applied to observe the residue in the furrow [27]. Before using the presentation, water washing and wet towel inside the forearm were imaged.

(4) VISIA-CR. Furthermore, to discover whether the lipstick and lip gloss can be removed with water or a wet towel on the lip, the VISIA-CR (Canfield Scientific, New Jersey, USA) was applied to image the lip [28]. Then, before the presentation, water washing and wet towel were imaged. Finally, the values of $a *$ and glossiness were measured using Image Plus Pro 7.0. 


\section{Results and Discussion}

3.1. Characterization of CNC-AN. Inspired by plant pigmentation as natural filters to protect themselves against overexposure to $\mathrm{UV}$, binding of $\mathrm{AN}$ to $\mathrm{CNC}$ was designed and prepared. Our previous study has applied CNC in foundation liquid [19]. Herein, we formulated traditional rouge from nontransparent minerals and plants such as clove and cinnabar (Figure 1(a)). We had been successfully attempted using either extracted AN from a variety of plants or commercialized AN (Supporting Information, Figure S1). The optimal ratio of AN and CNC for complex formation was determined (Supporting Information). The interaction between water-soluble AN and $\mathrm{CNC}$ occurred spontaneously and can bind to cellulose fibers up to $60 \% \mathrm{w} / \mathrm{w}$ (Figure S2). This finding suggests that the adsorption of AN on cellulose on plant cell walls can readily occur in real food systems whenever the plant tissues are processed or masticated [29]. The asprepared CNC-AN appeared as biomacromolecule particles whose average diameter was greater than $5 \mu \mathrm{m}$ as depicted in Figure 1(b) and Figure S3. AN acted as a layer of jelly covering the surfaces of CNC, showing strong binding interaction.

In addition, $\mathrm{AN}$ was complexed with $\mathrm{CNC}$ via hydrophilic, hydrogen, and electrostatic bonding interactions to form complexity (Figure 1(e)). There was sensitivity to native charges in the association between negatively charged cellulose fibers and positively charged AN [30]. Given this, zeta potential was approached. Zeta potential for CNC, $\mathrm{AN}$, and CNC-AN was determined to be $+7.87 \pm 0.65 \mathrm{mV}$, $-28.36 \pm 0.27 \mathrm{mV}$, and $-16.68 \pm 0.90 \mathrm{mV}$, respectively (Figure 1(c)). Additionally, the magnitude of negative zeta potential was increased by the addition of $\mathrm{CNC}$, thus improving AN electrostatic stabilization [31-33]. After grafting $\mathrm{AN}$ to $\mathrm{CNC}$, the bioadhesion of CNC-AN was clearly enhanced (Figure S4).

We further used FTIR to illustrate the type of molecular interactions between AN and CNC (Figure 1(d)). The characteristic peaks of CNC were around 3400, 2900, 1420 , and $1020 \mathrm{~cm}^{-1}$, which can be attributed to the $\mathrm{O}-\mathrm{H}$ stretching, C-H stretching, C-H bending, and C-O-C bending, respectively [34]. On the other hand, the characteristic peaks of AN were 1700,1400 , and $1190 \mathrm{~cm}^{-1}$, which can be attributed to the $\mathrm{C}=\mathrm{C}$ stretching vibration of the aromatic ring, C-O specific angular deformation, and $\mathrm{O}-\mathrm{C}$ stretching vibration of anhydroglucose ring, respectively [2]. Compared with $\mathrm{AN}$, we found no obvious change that occurred in the band of CNC-AN at $1700 \mathrm{~cm}^{-1}$, but CNC-AN became more intensive between 1000 and $1700 \mathrm{~cm}^{-1}$. These changes perhaps may be attributed to the stretching vibration of the $\mathrm{C}-\mathrm{C}$ bond of an aromatic ring in $\mathrm{AN}$ and the interactions between $\mathrm{AN}$ and CNC. Compared with $\mathrm{CNC}$, the intensity of the peak at $3400 \mathrm{~cm}^{-1}$ was significantly weakened. These changes are likely to be associated with the formation of a hydrogen bond between the free hydroxyl group. These findings implied that AN formed strong physical and chemical interactions with $\mathrm{CNC}[35,36]$.
3.2. Effect of CNC-AN on Specific Extinction Value, UV Absorption, and Shielding. To study the enhancing effects of UV absorbing properties of AN at different concentrations, we examined UV absorbing using a spectrophotometer. After binding $\mathrm{CNC}$ to commercialized $\mathrm{AN}$, we recorded a twofold increase in the UV absorption compared with AN alone (Figure 2(b)). Likewise, the extraction of the AN from purple cabbage showed similar results (Figure S5). However, our findings indicated no increase in UV absorption of benzophenone-3 (BP-3, a commercial sunscreen) with $\mathrm{CNC}$ (Figure S5). Ultrapure water and one common sun-screening agent (BP-3) served as a negative and positive control, respectively, while BP-3 was included as a positive control. We noticed that resonance delocalization in BP-3 was aided by the presence of an electron-releasing group in either the ortho position, para position, or both, which results in $\lambda$ max at $290 \mathrm{~nm}$ (Table S1 and Figure 2(b)). AN was composed of large $\pi$-conjugated domains with the formation of aromatic structures, which the maximum absorption wavelength $(\lambda \max )$ was characterized at $280 \mathrm{~nm}$ (Table S1 and Figure 2(b)).

Furthermore, one challenge here was that naturally sourced UV filter application to sunscreen did not achieve a desired sun protection factor (SPF) value because of the low specific extinction value $(\mathrm{E} 1,1)$. As UV-filters, the specific extinction value $(\mathrm{E} 1,1)$ is more important than UVabsorbance. We obtained the extinction E1,1 ( $\lambda \max )$ using the following equation [16].

$$
\mathrm{E} 1,1(\lambda)=\varepsilon(\lambda) \frac{10}{M} \bullet d
$$

where $\varepsilon$ refers to the molar decadic extinction coefficient, $M$ is the molecular weight, and $d$ denotes the optical pathlength. The extinction coefficient $\varepsilon$ was calculated from the transmittance measurements according to the following equation.

$$
E(\lambda)=\log \frac{1}{T(\lambda)},
$$

where $T$ refers to the transmittance.

$$
\varepsilon(\lambda)=\frac{E(\lambda)}{c \bullet d},
$$

where $E$ denotes the extinction calculated via Eq, $\mathrm{c}$ in concentration in $\mathrm{mol} \cdot \mathrm{L}^{-1}$ and $d$ is the optical pathlength in $\mathrm{cm}$.

Our findings elucidated that the difference between BP-3 and $\mathrm{AN}$ in the E1,1 $(\lambda \max )$ value was about a 5 -fold improvement (Table S1 and Figure 2(c)). Of note, E1,1 $(\lambda \max )$ presented the extinction E1,1 maximum wavelength, whereas $\lambda$ max presented the wavelength of maximum absorbance. Elsewhere, both $\mathrm{E} 1,1(\lambda \max )$ and $\lambda \max$ are two prime parameters that are identified to describe the performance of a UV-filter molecule [16].

In addition, using a UV light and a UV irradiance meter, the UV-shielding efficiencies of AN, CNC, CNC-AN, and 


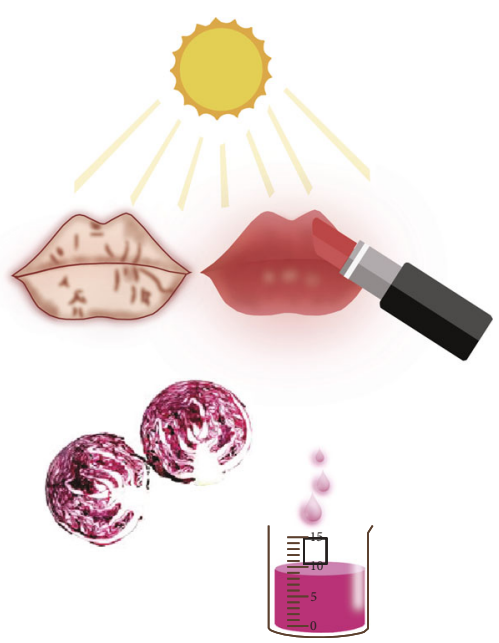

(a)

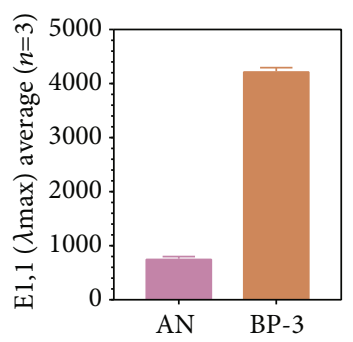

(c)

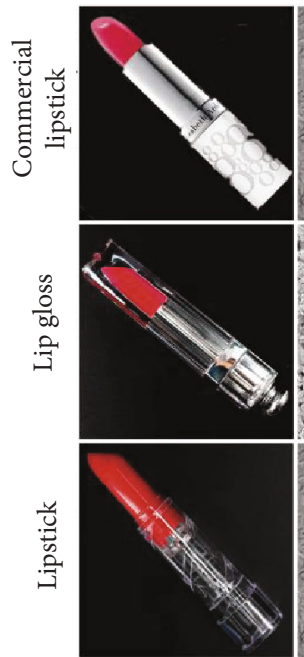

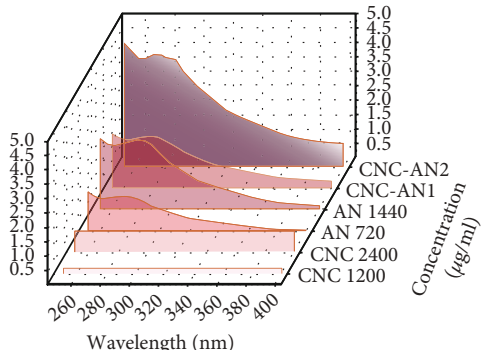

(b)

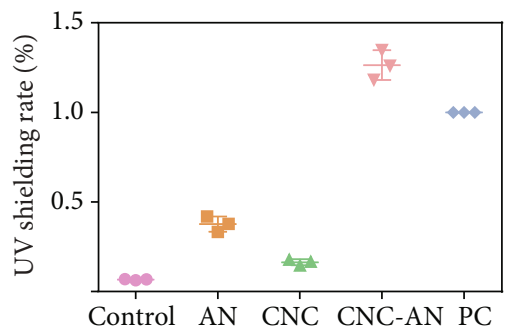

(d)

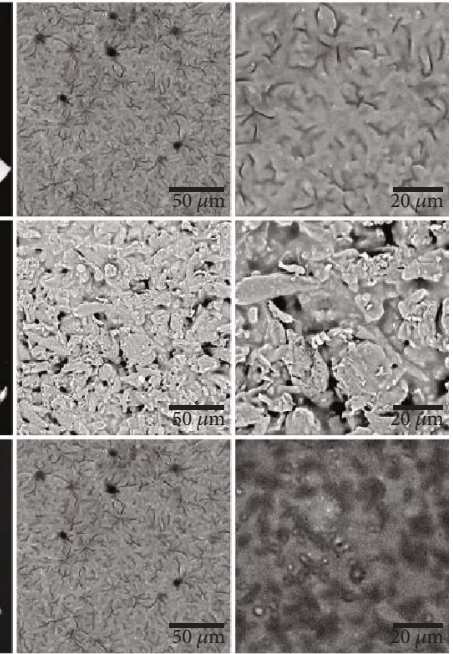

(e)

\begin{tabular}{cccc}
\hline & Lip gloss & Lipstick & Commercial lipstick \\
\hline SPF & $22.12 \pm 1.58$ & $30.33 \pm 2.77$ & $12.22 \pm 0.23$ \\
\hline
\end{tabular}

(f)

Figure 2: (a) Schematic design concept of sunscreen lipsticks. (b) The UV absorption of CNC, AN, and CNC-AN. (c) E1,1 ( $\lambda$ max) value of BP-3 and AN. (d) UV shielding rate of CNC, AN, CNC grafting AN, and BP-3 as a positive control. (e) Scanning electron microscopic (SEM) images of lip gloss, lipstick, and commercial lipstick. (f) Values of SPF of lip gloss, lipstick, and commercial lipstick.

BP-3 with $15 \mu \mathrm{m}$ thickness were irradiated. Our results uncovered that the standard sunscreen agent BP-3 was considered as $100 \%$ UV shielding, while that of CNC-AN homogenate was higher compared with the positive control, showing efficiency absorption $>126 \%$ UV light. Finally, after grafting $\mathrm{CNC}$ to $\mathrm{AN}$, the $\mathrm{UV}$ shielding rate of the coupled AN tripled that of AN alone (Figure 2(d)).

3.3. Preparation and Characterization of Lipstick. Based on the improved UV shielding rate and ultraviolet absorption, 
we used CNC-AN homogenate to formulate sunscreen lipstick (Figure 2(e) and Table S2). Briefly, the homogenate was freeze-dried into a powder that was applied to formulate CNC-AN lipstick (commercial lipstick as positive control) (Figure 2(e)). Afterward, we herein employed two kinds of lip products, including cationic hydroxyethyl cellulose (cHEC) and diverse oils, to prepare the CNC-AN formula, and their resulting products were termed lipstick and lip gloss, respectively. In particular, lipstick was composed of CNC-AN (1 g), soft oil (6.6 g), and hard wax (2.1 g), as consistency in the traditional formula, while lip gloss comprised of CNC-AN complexed with cHEC hydrogels via hydrophilic, hydrogen bonding, and/or electrostatic interactions.

Then, the stability of formulations (lipstick/lip gloss) was studied. Both lipstick and lip gloss had red color. The $\mathrm{pH}$ of lipstick and lip gloss was range from 5 to 5.5 which were suitable for topical application. The result of stability studies showed that lipstick and lip gloss were stable during 3 months. The layering phenomenon was not present in lip gloss after returning to room temperature. The lipstick stayed hard after returning to room temperature.

The microscopic hierarchical structures of lip gloss, lipstick, and commercial lipstick were examined under a scanning electron microscope (SEM) (Figure 2(e)). The results highlighted that commercial lipstick exhibited a smooth structure, whereas lip gloss and lipstick containing CNC showed granular sensation (Figure 2(e)). The convex and flat profile belonged to the connection of CNC-AN to the substrate, yielding substantial interfacial adhesion. The $\mathrm{TiO}_{2}$ fillers were visible in commercial lipstick. Recent studies have established that long-term consumption of $\mathrm{TiO}_{2}$ is harmful to the body, such as disturbance of gut microbiota, intestinal inflammation, and immune response [37]. We also noted that lip gloss possesses lamellar microstructures with heterogeneity, while in lipstick, even fine particles dispersed.

\subsection{Effect of Lipstick on In Vitro SPF. SPF value was detected} using SPF $290 \mathrm{~S}\left(2 \mathrm{mg} / \mathrm{cm}^{2}\right.$ on $3 \mathrm{M}$ film). The optometric model SPF-290 analyzer is a computer-controlled instrument that is designed to measure the SPF value of sunscreen preparations based on the US-FDA standards [38]. The collected data were plotted as an MPF (monochromatic protection factor) [38]. Our results of the SPF presented a very good correlation with the BASF sunscreen simulator presented, which exhibited a good correlation with real-world figures in vivo [38]. The SPF revalidated with 5\% homosalate (HMS), 5\% octocrylene (OCR), 4\% 4-methylbenzylidene camphor (MBC), and 5\% BP-3 hydroxyethyl cellulose hydrogel that are summarized in Table S3. Moreover, the values of SPF were $22.12 \pm 1.58$ for lip gloss, lipstick $(30.33 \pm 2.77)$, and commercial lipstick (12.22 \pm 0.23$)$, respectively (Figure 2(f)). These results suggest that lip gloss and lipstick possess high protection levels (usually corresponding to a measured SPF value $>15$ for lipstick) as potential sunscreens [39]. Notably, the SPF value was improved 10-folder higher absorption after grafting CNC to AN compared with the AN alone. However, a similar amplification effect was not observed with chemical UV- filters. Overall, it is important to note that CNC can significantly improve the anti-UVB function of AN.

3.5. Bioadhesive Characteristics and Retention of Lipstick. We directly applied fluorescein isothiocyanate (FITC) to these samples by rubbing on the dorsal skin of nude mice, followed by imaging using an in vivo imaging system (IVIS). We observed no significant change in the fluorescent intensity among the three groups at $0,2,4$, and $6 \mathrm{~h}$ (Figures $3(\mathrm{a})$ and $3(\mathrm{~d})$ ), revealing that the excellent bioadhesion and retention of lip gloss and lipstick were inconsistent with commercial lipstick. Likewise, the water resistance of the lip gloss and lipstick was evaluated using IVIS. The fluorescent intensity exhibited no change after water washing among the three groups, indicating good resistance against water. However, the lip gloss and lipstick were easy to wipe with a wet towel, while the commercial lipsticks were difficult to wipe (Figures 3(b) and 3(e)). Remarkably, easy wiping with a wet towel can decrease the use of facial cleanser, cleansing water, and cleansing oil, which is friendly to the skin particularly for those people with skin diseases [40, 41]. Furthermore, to identify the penetration behaviors, free FITC, lip gloss, and lipstick were applied to the pigskin, which was used as a surrogate for human skin [42]. After $6 \mathrm{~h}$, the pigskin samples were frozen in a frozen section compound, and fluorescent depth was recorded (Figures 3(c) and $3(f))$. We noted that free FITC penetrated the dermis, while lip gloss and lipstick did not penetrate the skin. These findings signify biosafety which is critical in the cosmetic industry [43]. Therefore, all characteristics including excellent bioadhesion, water resistance, easy wiping with a wet towel, and no penetration make CNC-AN gel and lipstick better products in the cosmetic industry.

3.6. Evaluation of Anti-UVB-Induced Oxidative and DNA Damage Activity In Vitro. To evaluate the safety of CNCAN freeze-dried powder, we herein employed a cell viability assay which was detected using CCK-8 assay. Up to $10 \mathrm{mg} /$ $\mathrm{ml}$, the number of cells showed no decline at all. However, the number of cells improved compared to the control group (Figure S6). This outcome illustrates that CNC-AN freezedried powder did not influence the viability of $\mathrm{HaCaT}$ cells, which shows assured biosecurity. Using ROS level, comet assay, and cell immunofluorescence in vitro, we also demonstrated the protection of UVB-induced damage in lip gloss and lipstick. In particular, one kind of common sunscreen (product name: Neutrogena SPF 30 Dry-touch Sunscreen) was used as a positive control ( $\approx 15 \mu \mathrm{m}$ thickness). The level of ROS was detected as green fluorescence proportional intensity. After UVB irritation, the intracellular ROS level in HaCaT cells was significantly different compared with control, sunscreen, lip gloss, and lipstick groups. The ROS level in the lip gloss and lipstick groups showed no significant differences compared to the control and sunscreen groups, suggesting that CNC-AN, lip gloss, and lipstick can protect cells against UVB-induced oxidative stress (Figure S7). Then, we measured the single and double-strand break of DNA in cells using the comet assay [22]. The percentage of DNA content in the comet tail might represent the levels 


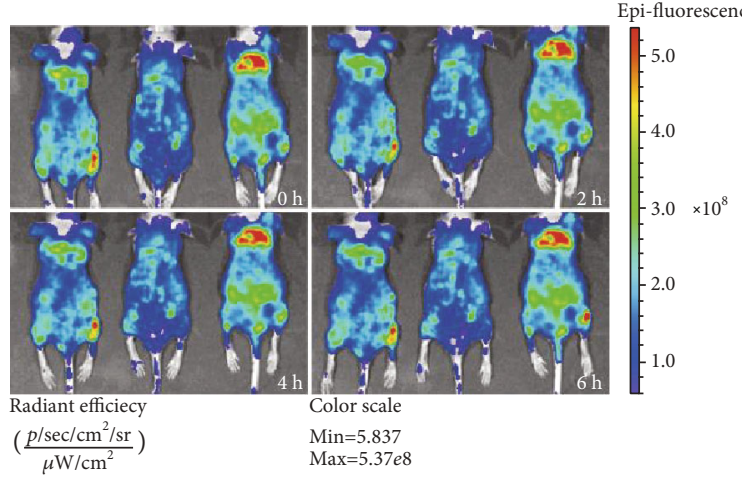

(a)

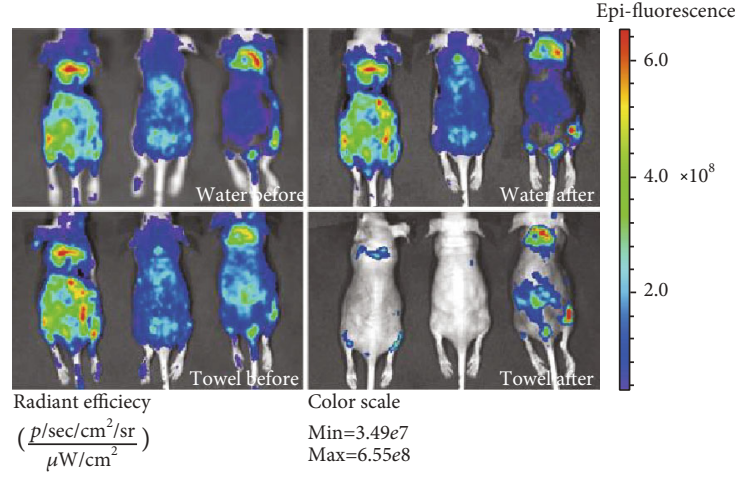

(b)

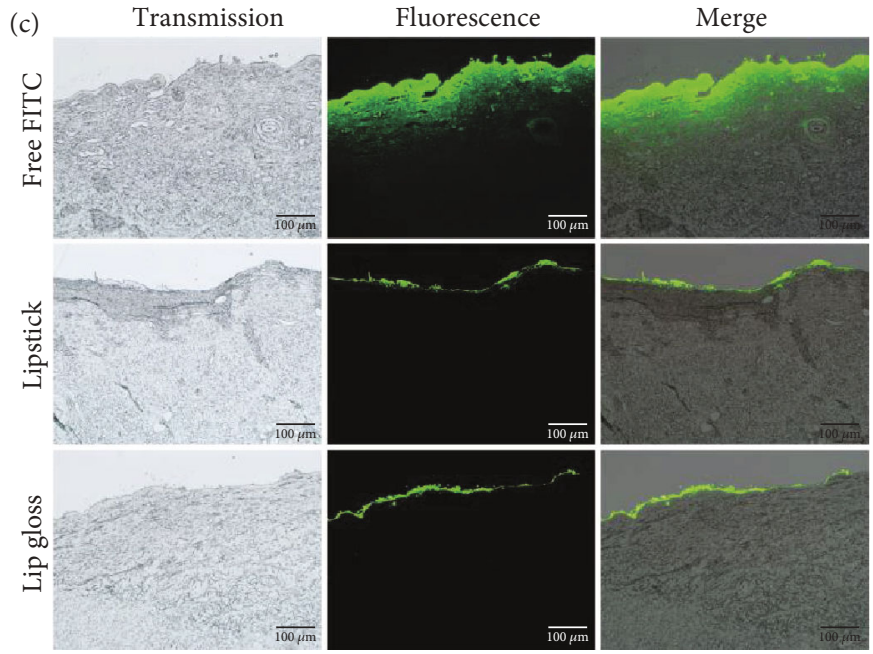

(c)

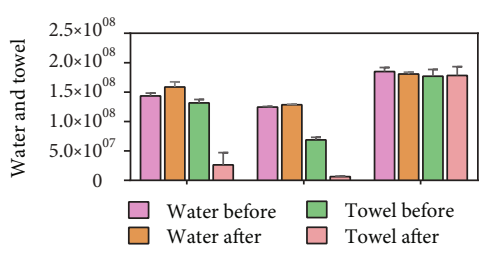

(e)

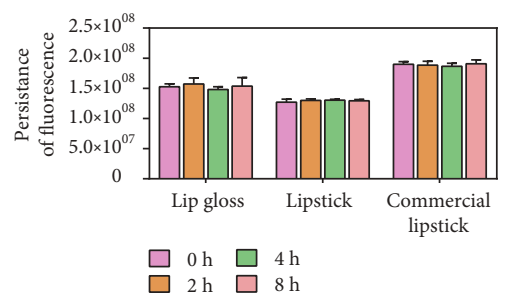

(d)

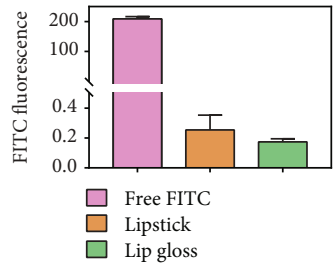

(f)

FIGURE 3: (a) IVIS images of the bioadhesion and retention of lip gloss, lipstick, and commercial lipstick at 0, 2, 4, and $6 \mathrm{~h}$. (b) IVIS images of free FITC, lip gloss, and lipstick cleansing after washing with water or wiping with a wet towel. (c) Transdermal penetration of free FITC, lip gloss, and lipstick in pigskin. (d) The fluorescence intensity of bioadhesive and retention in lip gloss, lipstick, and commercial lipstick. (e) The fluorescence intensity of cleansing water and towel in lip gloss, lipstick, and commercial lipstick. (f) The penetration depth of free FITC, lip gloss, and lipstick.

of DNA damage. Specifically, DNA\% tail was higher in the UVB group, while lip gloss and lipstick groups exhibited no change compared with the control and sunscreen groups (Figures $4(\mathrm{a})$ and $4(\mathrm{~b})$ ). $\gamma \mathrm{H} 2 \mathrm{AX}$ is a marker of nucleus damage [44]. Subsequently, we applied cell immunofluorescence to detect the expression of $\gamma \mathrm{H} 2 \mathrm{AX}$ in order to demonstrate the protection of CNC-AN gel and lipstick from UVBinduced nucleus impairment. It is apparent that while the expression of $\gamma \mathrm{H} 2 \mathrm{AX}$ improved a lot in the UVB group, the lip gloss and lipstick groups showed no differences compared with the control and sunscreen groups (Figures 4(c)- 4(f)). From comet assay and cell immunofluorescence $\mathrm{H} 2 \mathrm{AX}$, the protection of nucleus damage was evident.

3.7. Evaluation of Anti-UVB Damage Activity In Vivo. We further performed protection in vivo research to observe the efficiency of CNC-AN, lip gloss, and lipstick in Balb/c mice. We divided the back of these mice into 4 sections, including control, UVB, sunscreen, and experimental groups (Figure 5(a)). One of them was dealt with gel, while another was dealt with lipstick in the experimental group (every section was covered with $1 \mathrm{~cm} \times 1 \mathrm{~cm} \times 100 \mu \mathrm{m}$ sunscreen, lip 


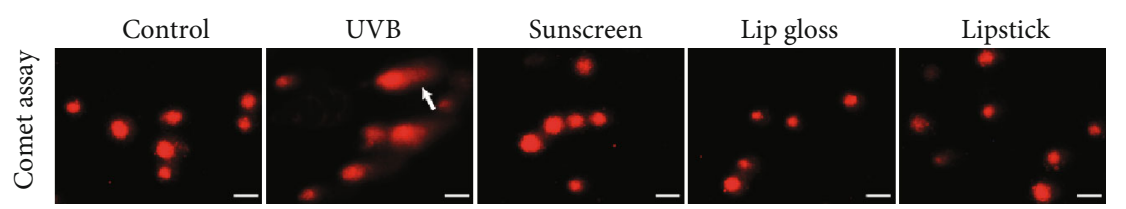

(a)
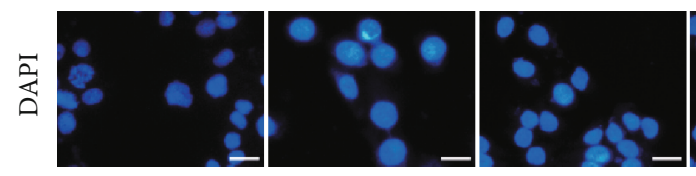

(c)
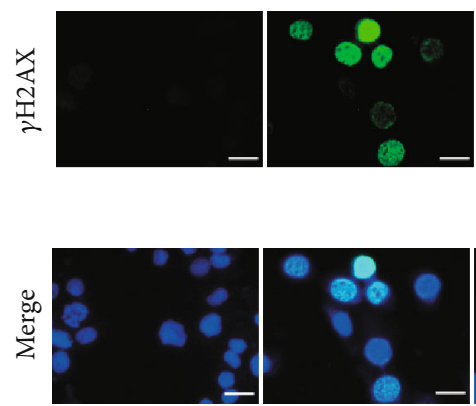

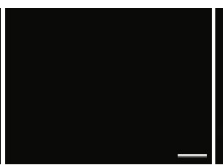

(d)

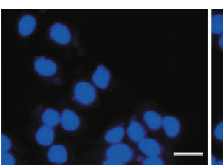

(e)

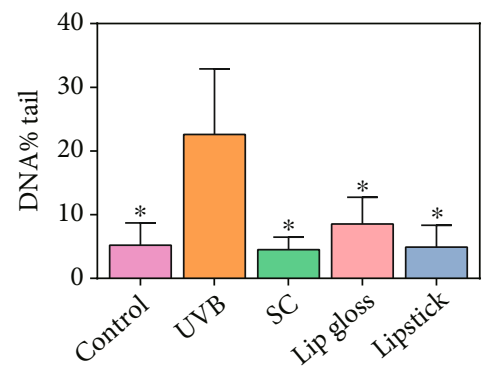

(b)

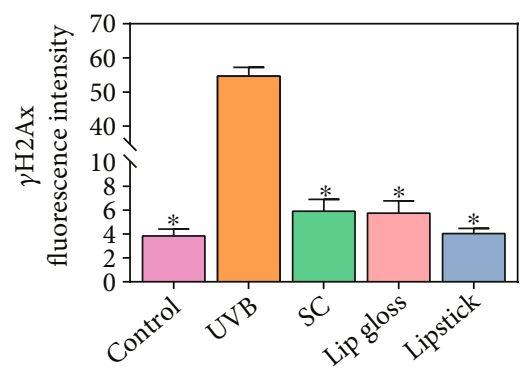

(f)

Figure 4: (a) The comet assay was applied to detect the DNA damage in HaCaT cells. (b) The statistical figure of DNA\% tail. (c-e) $\gamma \mathrm{H} 2 \mathrm{AX}$ cell immunofluorescence was applied to evaluate the nucleus damage. (f) The statistical figure of $\mathrm{H} 2 \mathrm{AX}$ fluorescence level. ${ }^{*} P<0.05$, the difference has statistical significance. Scale bars, $50 \mu \mathrm{m}$.

gloss, and lipstick, except the control and UVB groups. In addition, the control group was covered with a silver paper). These mice were exposed to UVB radiation with $5040 \mathrm{~mJ} /$ $\mathrm{cm}^{2}$ for single use. After radiation, the dermatoscope and hematoxylin/eosin staining of dorsal skin in each group was executed at 24, 48, and $72 \mathrm{~h}$, respectively (Figures 5(b) and 5(c), S8, S9). In the images of the dermatoscope, UVBinduced damage in the skin after 24 and $48 \mathrm{~h}$ was illegible (Figure S8). However, after $72 \mathrm{~h}$, the UVB group manifested clearly erythema, blister, erosion, and crust. Notably, the erythema value in the UVB group at $72 \mathrm{~h}$ was much higher than in other groups (Figure 5(b)). The CNC-AN gel and lipstick group conformed to the control and sunscreen groups, which identified the efficient protection against UVB. Based on the hematoxylin/eosin staining of the dorsal skin, the thickness of the skin was increased clearly at 24 and $48 \mathrm{~h}$ in the UVB group compared with other groups (Figure S9). In addition, the dermis was fully inflammatory infiltrate while sebaceous structures looked hypertrophic, especially at $72 \mathrm{~h}$ in the UVB group. Furthermore, the skin disappeared completely at $72 \mathrm{~h}$ after UVB radiation, with alteration of the skin replaced by blisters and crust (Figures 5(b) and 5(c)). Similarly, the protection of the CNC-AN, lip gloss, and lipstick groups showed no substantial change relative to the control and sunscreen groups.

Moreover, the epidermal and sebaceous hypertrophy effect in the skin protected by CNC-AN, lip gloss, and lipstick was significantly inhibited even compared with the sunscreen group, which appeared slightly hypertrophic relative to the control group. In this study, we applied lip culture in BALB/C mice so as to confirm the protection of CNCAN, lip gloss, and lipstick for lips. To begin with, lip explants were incubated epidermal-side-up on a transwell in 6-well plates. Briefly, 5 wells were assigned to control, UVB, sunscreen, lip gloss, and lipstick groups, respectively (Figure 5(d)). Every well was covered with $100 \mu \mathrm{m}$ sunscreen, lip gloss, and lipstick, except for the control and UVB groups. The control group was covered with a silver paper. Then, a 6-well plate was exposed to UVB radiation with $5040 \mathrm{~mJ} / \mathrm{cm}^{2}$ for single use. After radiation, owing to evidence from dorsal skin, hematoxylin/eosin staining of the lip in each group was performed at $72 \mathrm{~h}$ (Figure 5(e)). The thickness of the lip increased apparently at $72 \mathrm{~h}$ in the UVB group relative to other groups. Interestingly, the protection of the CNC-AN gel and lipstick group showed significant protection compared with the control and sunscreen groups. Both organotypic cultures and in vivo experiments manifested excellent anti-UVB protection of CNC-AN gel and lipstick.

3.8. Human Evaluation. The bioadhesion and retention of CNC-AN lip gloss and lipstick in human skin were examined using CIE 3D space system. After application, the changes in $L *$ and $a *$ values for $8 \mathrm{~h}$ were measured at 9 time points. There were no significant differences in the values of $L *$ and $a *$ among lip gloss, lipstick, and commercial lipsticks, showing excellent bioadhesion and retention in agreement with commercial lipstick (Figure S11). To explore the water persistence and easy wiping with a wet towel of CNC-AN gel and lipstick in the human lip, VISIA was 


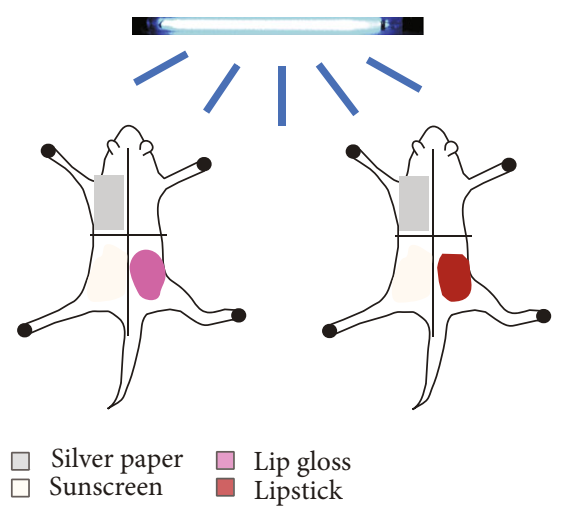

(a)
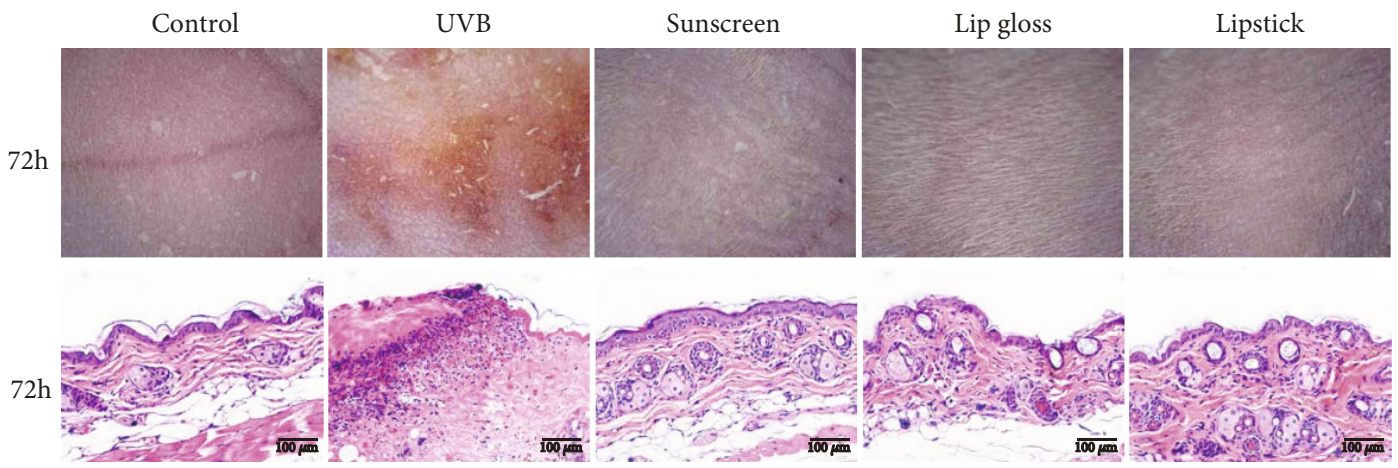

(b)

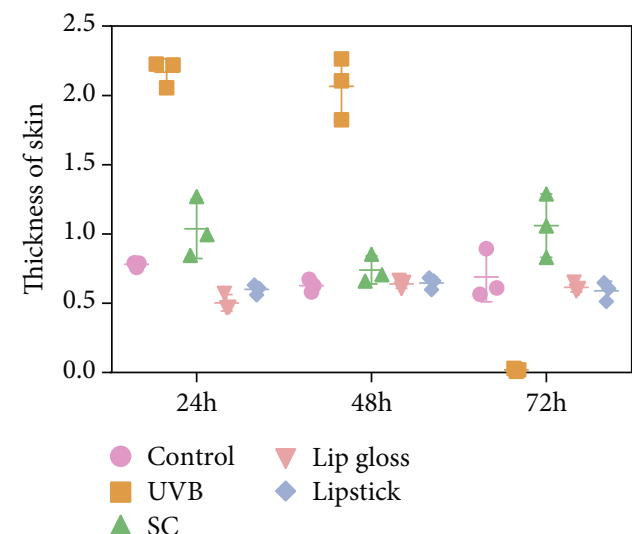

(c)

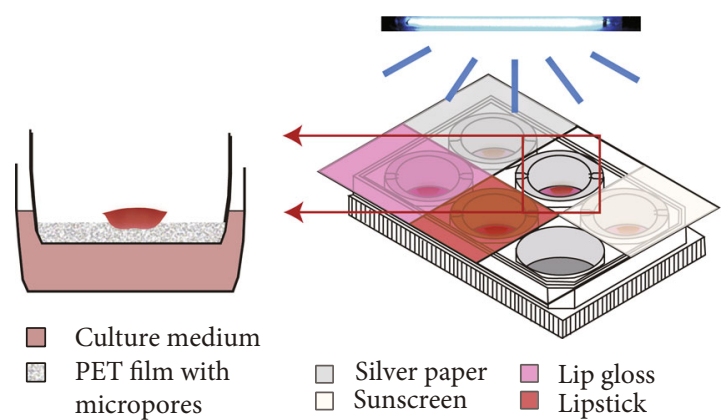

(d)

Control UVB

Sunscreen Lip gloss Lipstick

$72 \mathrm{~h}$

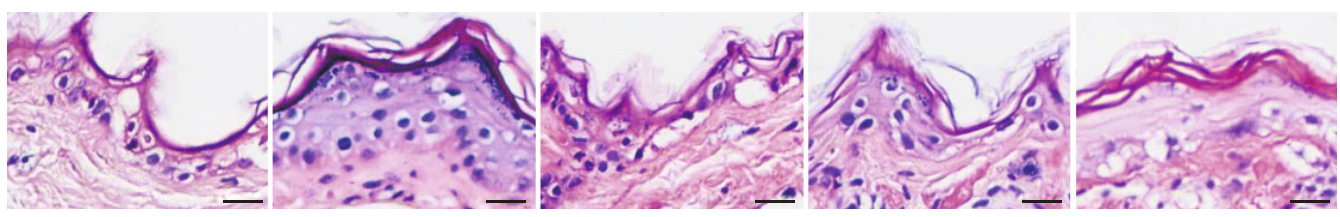

(e)

Figure 5: Continued. 


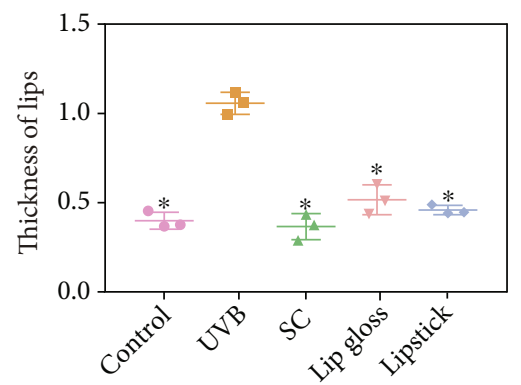

(f)

FIgURE 5: Dermatoscope and histology of dorsal mouse skin applying different topical interventions after UV irradiation. (a) Model diagram of mouse protection against UVB irradiation. (b) The dermatoscope and hematoxylin/eosin staining of dorsal skin in mice. (c) Thickness of skin in mice. ${ }^{*} P<0.05$, the difference has statistical significance. Scale bars, $100 \mu \mathrm{m}$. (d) Model diagram of organotypic culture showing lip protection against UVB irradiation. (e) Hematoxylin/eosin staining of the lip in each group. ${ }^{*} P<0.05$, the difference has statistical significance. Scale bars, $25 \mu \mathrm{m}$.
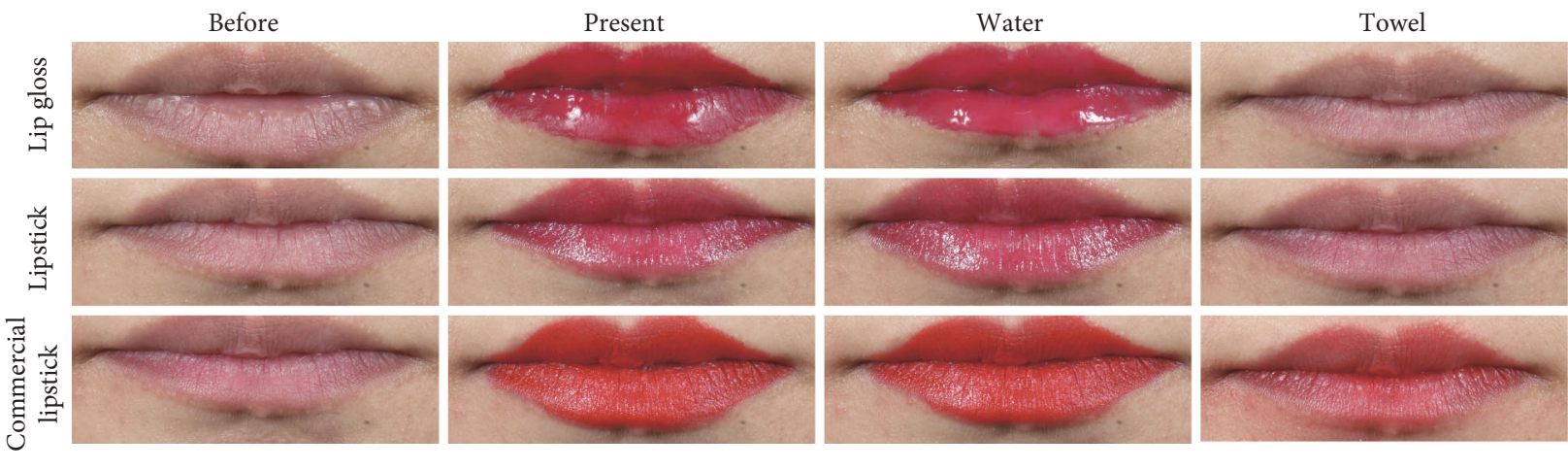

(a)
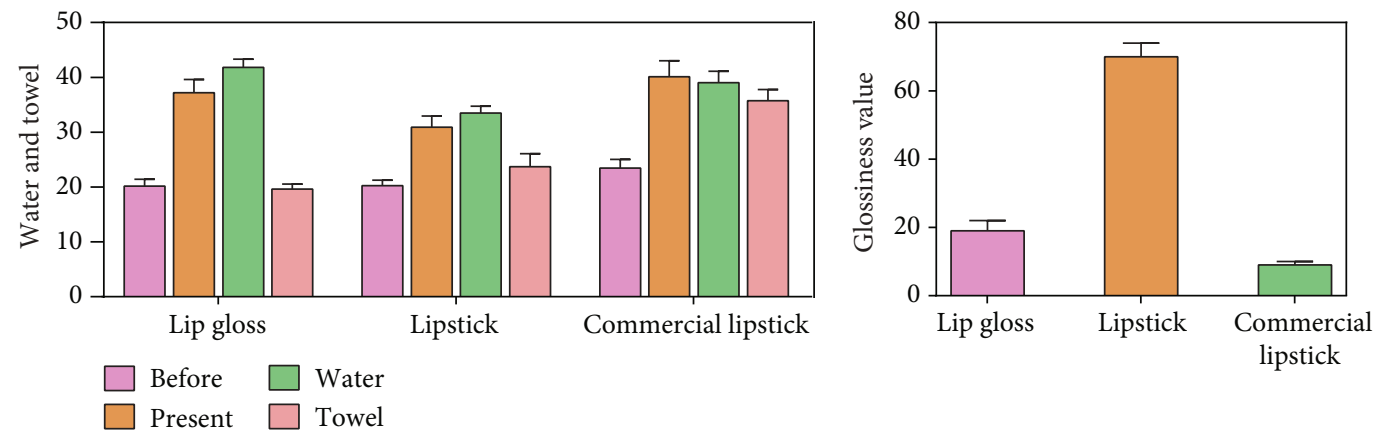

(b)

(c)

FIGURE 6: (a) VISIA images of lip gloss, lipstick, and commercial lipstick cleansing after washing with water or wiping with a wet towel. (b) The values of VISIA images. (c) The glossiness value before and after applying lipsticks.

applied to collect photographs at different time points (Figure 6(a)). We recorded the glossiness value before and after applying lip gloss, lipstick, and commercial lipstick. Our findings revealed that CNC-AN gel and lipstick were better than commercial lipstick (Figure 6(c)). After water washing, all of these three gel and lipsticks were not removed, thus indicating excellent water persistence. Further confirmation, after cleaning with a wet towel, lip gloss and lipstick groups were completely discarded, while the commercial lipstick group left a large amount of residual lipstick on the lips (Figure 6(b)). The characteristics of water persistence and easy wiping with a wet towel were also proved by the use of a dermatoscope (Figure S10). In conclusion, the characteristics of wonderful bioadhesion and water persistence are qualified for a product as lipstick. Furthermore, after applying the $\mathrm{CNC}$, the gel and lipstick convert to easy wiping with a wet towel, offering those people with skin diseases, such as rosacea, contact dermatitis, acne, and some other sensitive skin that are too fragile to use a facial cleanser. Finally, it is convenient to clean it at any time such 
as before meals, for the reason that lipstick eating with food is harmful to health [45].

\section{Conclusion}

In summary, the protection of UV-induced lip damage has recently attracted greater research attention. Inspired by the traditional Chinese method, we applied anthocyanins to formulate lipsticks. Combining our previous research, we grafted CNC with AN to form a biomolecule characterized by enhanced bioadhesion, retention, and easy wiping characteristics. Surprisingly, CNC improved the SPF value of our new lipstick beyond our expectation. Therefore, our new creativeness provides a series of natural-sourced, environmentally friendly raw materials for more widespread use in the cosmetic industry. Finally, our lipstick has potential for commercial application owing to its natural-sourced, environmentally friendly, excellent bioadhesion, water resistance, easy wiping with a wet towel, and excellent UV-shielding features that enable this sunscreen lipstick to fill a gap in the market.

\section{Data Availability}

The data used to support the findings of this study are included within the article and supplementary information files.

\section{Additional Points}

Rationale. Cellulose nanocrystals reinforced enhancement of UV absorption and shielding properties of anthocyanin by improving specific extinction value. Objective. Safer biobased sunscreens of cellulose enveloped with anthocyanin were bionic designed evaluated in vitro and in vivo.

\section{Conflicts of Interest}

The authors declare no conflict of interest.

\section{Authors' Contributions}

Lidan Xiong and Hailun He are co-first authors.

\section{Acknowledgments}

This work was supported by 1.3 .5 project for disciplines of excellence, West China Hospital, Sichuan University, and China National Natural Science Foundation (No. 81673084).

\section{Supplementary Materials}

The supplementary description includes graph abstract of our research, the method for preparation and characterization of anthocyanin, binding capacity experiments, scanning electron microscopic (SEM) images of CNCs and CNCs grafting $\mathrm{AN}$, continuous shooting of $\mathrm{CNCs}, \mathrm{AN}$ and CNCs-AN, UV absorption, cell viability assay, ROS fluorescence level, dermatoscope and H\&E staining of mouse dorsal skin, dermatoscope, and CIE 3D in forearm skin in human. (Supplementary Materials)

\section{References}

[1] L. Baker, B. Marchetti, T. Karsili, V. Stavros, and M. Ashfold, "Photoprotection: extending lessons learned from studying natural sunscreens to the design of artificial sunscreen constituents," Chemical Society Reviews, vol. 46, no. 12, pp. 37703791, 2017.

[2] K. Zhang, T. S. Huang, H. Yan, X. Hu, and T. Ren, "Novel pHsensitive films based on starch/polyvinyl alcohol and food anthocyanins as a visual indicator of shrimp deterioration," International Journal of Biological Macromolecules, vol. 145, pp. 768-776, 2020.

[3] R. C. Lundeen, R. P. Langlais, and G. T. Terezhalmy, "Sunscreen protection for lip mucosa: a review and update," Journal of the American Dental Association (1939), vol. 111, no. 4, pp. 617-621, 1985.

[4] J. M. Pogoda and S. Preston-Martin, "Solar radiation, lip protection, and lip cancer risk in Los Angeles County women (California, United States)," Cancer Causes \& Control, vol. 7, no. 4, pp. 458-463, 1996.

[5] I. Rodriguez-Blanco, A. Florez, C. Paredes-Suarez et al., "Use of lip photoprotection in patients suffering from actinic cheilitis," European Journal of Dermatology, vol. 29, no. 4, pp. 383386, 2019.

[6] A. Agrawal, E. Shindell, F. Jordan, L. Baeva, J. Pfefer, and D. E. Godar, "UV radiation increases carcinogenic risks for oral tissues compared to skin," Photochemistry and Photobiology, vol. 89, no. 5, pp. 1193-1198, 2013.

[7] J. B. Mancuso, R. Maruthi, S. Q. Wang, and H. W. Lim, "Sunscreens: an update," American Journal of Clinical Dermatology, vol. 18, no. 5, pp. 643-650, 2017.

[8] S. Q. Wang, M. E. Burnett, and H. W. Lim, "Safety of oxybenzone: putting numbers into perspective," Archives of Dermatology, vol. 147, no. 7, pp. 865-866, 2011.

[9] S. M. Herzog, H. W. Lim, M. S. Williams, I. D. de Maddalena, U. Osterwalder, and C. Surber, "Sun protection factor communication of sunscreen effectiveness: a web-based study of perception of effectiveness by dermatologists," JAMA Dermatology, vol. 153, no. 3, pp. 348-350, 2017.

[10] Y. Deng, A. Ediriwickrema, F. Yang, J. Lewis, M. Girardi, and W. M. Saltzman, "A sunblock based on bioadhesive nanoparticles,” Nature Materials, vol. 14, no. 12, pp. 1278-1285, 2015.

[11] M. Matta, J. Florian, R. Zusterzeel et al., "Effect of sunscreen application on plasma concentration of sunscreen active ingredients: a randomized clinical trial," JAMA, vol. 323, no. 3, pp. 256-267, 2020.

[12] Y. Wu, M. Venier, and R. Hites, "Broad exposure of the North American environment to phenolic and amino antioxidants and to ultraviolet filters," Environmental Science \& Technology, vol. 54, no. 15, pp. 9345-9355, 2020.

[13] K. Tsoyi, H. Park, Y. Kim et al., "Anthocyanins from black soybean seed coats inhibit UVB-induced inflammatory cylooxygenase-2 gene expression and PGE2 production through regulation of the nuclear factor-kappaB and phosphatidylinositol 3-kinase/Akt pathway," Journal of Agricultural and Food Chemistry, vol. 56, no. 19, pp. 8969-8974, 2008.

[14] I. Choi, J. Y. Lee, M. Lacroix, and J. Han, "Intelligent pH indicator film composed of agar/potato starch and anthocyanin extracts from purple sweet potato," Food Chemistry, vol. 218, pp. 122-128, 2017.

[15] M. Pandika, "Looking to nature for new sunscreens," ACS Central Science, vol. 4, no. 7, pp. 788-790, 2018. 
[16] M. Sohn, L. Amorós-Galicia, S. Krus, K. Martin, and B. Herzog, "Effect of emollients on UV filter absorbance and sunscreen efficiency," Journal of Photochemistry and Photobiology. B, vol. 205, article 111818, 2020.

[17] P. Ric-Varas, M. Barceló, J. Rivera et al., "Exploring the use of fruit callus culture as a model system to study color development and cell wall remodeling during strawberry fruit ripening," Plants, vol. 9, no. 7, p. 805, 2020.

[18] B. Herzog, K. Quass, E. Schmidt, S. Müller, and H. Luther, "Physical properties of organic particulate UV absorbers used in sunscreens: II. UV-attenuating efficiency as function of particle size. UV-attenuating efficiency as function of particle size," Journal of Colloid and Interface Science, vol. 276, no. 2, pp. 354-363, 2004.

[19] J. Tang, H. He, R. Wan et al., "Cellulose nanocrystals for skin barrier protection by preparing a versatile foundation liquid," ACS omega, vol. 6, no. 4, pp. 2906-2915, 2021.

[20] L. Kang, P. Chen, B. Wang et al., "Cellulose nanocrystal dye as reinforcement matrix of lipstick for inhibiting color migration," Cellulose, vol. 27, no. 2, pp. 905-913, 2020.

[21] P. Choochana, J. Moungjaroen, N. Jongkon, W. Gritsanapan, and P. Tangyuenyongwatana, "Development of piperic acid derivatives from Piper nigrum as UV protection agents," Pharmaceutical Biology, vol. 53, no. 4, pp. 477-482, 2015.

[22] H. He, J. Tang, D. Ru et al., "Protective effects of Cordyceps extract against UVB-induced damage and prediction of application prospects in the topical administration: An experimental validation and network pharmacology study," Biomedicine \& Pharmacotherapy, vol. 121, article 109600, 2020.

[23] R. J. Goodwin, J. C. Dungworth, S. R. Cobb, and A. R. Pitt, "Time-dependent evolution of tissue markers by MALDI-MS imaging," Proteomics, vol. 8, no. 18, pp. 3801-3808, 2008.

[24] X. Lin, Z. Wang, H. Ou, S. Mitragotri, and M. Chen, "Correlations between skin barrier integrity and delivery of hydrophilic molecules in the presence of penetration enhancers," Pharmaceutical Research, vol. 37, no. 6, p. 100, 2020.

[25] M. M. Nielsen, J. D. Schmidt, J. P. Christensen, C. Geisler, J. D. Johansen, and C. M. Bonefeld, "Detection of local inflammation induced by repeated exposure to contact allergens by use of IVIS SpectrumCT analyses," Contact Dermatitis, vol. 76, no. 4, pp. 210-217, 2017.

[26] V. Honson, Q. Huynh-Thu, M. Arnison, D. Monaghan, Z. J. Isherwood, and J. Kim, "Effects of shape, roughness and gloss on the perceived reflectance of colored surfaces," Frontiers in Psychology, vol. 11, p. 485, 2020.

[27] E. Muroi, T. Hara, K. Yanaba et al., "A portable dermatoscope for easy, rapid examination of periungual nailfold capillary changes in patients with systemic sclerosis," Rheumatology International, vol. 31, no. 12, pp. 1601-1606, 2011.

[28] J. D. Holcomb, "Helium plasma dermal resurfacing: VISIA CR assessment of facial spots, pores, and wrinkles-preliminary findings," Journal of Cosmetic Dermatology, vol. 20, no. 6, pp. 1668-1678, 2021.

[29] A. D. Phan, G. Netzel, D. Wang, B. M. Flanagan, B. R. D'Arcy, and M. J. Gidley, "Binding of dietary polyphenols to cellulose: structural and nutritional aspects," Food Chemistry, vol. 171, pp. 388-396, 2015.

[30] M. C. Smith, R. M. Crist, J. D. Clogston, and S. E. McNeil, "Zeta potential: a case study of cationic, anionic, and neutral liposomes," Analytical and Bioanalytical Chemistry, vol. 409, no. 24, pp. 5779-5787, 2017.
[31] N. Nirmalkar, A. Pacek, and M. Barigou, "On the existence and stability of bulk nanobubbles," Langmuir : the ACS journal of surfaces and colloids, vol. 34, no. 37, pp. 10964-10973, 2018.

[32] S. Fuziwara, K. Ogawa, D. Aso, D. Yoshizawa, S. Takata, and M. Denda, "Barium sulphate with a negative $\zeta$ potential accelerates skin permeability barrier recovery and prevents epidermal hyperplasia induced by barrier disruption," The British Journal of Dermatology, vol. 151, no. 3, pp. 557-564, 2004.

[33] P. Lauren, H. Paukkonen, T. Lipiainen et al., "Pectin and mucin enhance the bioadhesion of drug loaded nanofibrillated cellulose films," Pharmaceutical Research, vol. 35, no. 7, p. 145, 2018.

[34] Y. Liu, D. Ying, L. Sanguansri, Y. Cai, and X. Le, "Adsorption of catechin onto cellulose and its mechanism study: kinetic models, characterization and molecular simulation," Food Research International, vol. 112, pp. 225-232, 2018.

[35] H. Lee, S. M. Dellatore, W. M. Miller, and P. B. Messersmith, "Mussel-inspired surface chemistry for multifunctional coatings," Science (New York, N.Y.), vol. 318, no. 5849, pp. 426430, 2007.

[36] S. Zhou, X. Kong, B. Zheng, F. Huo, M. Strømme, and C. Xu, "Cellulose nanofiber@ conductive metal-organic frameworks for high-performance flexible supercapacitors," ACS Nano, vol. 13, no. 8, pp. 9578-9586, 2019.

[37] W. Mu, Y. Wang, C. Huang et al., "Effect of long-term intake of dietary titanium dioxide nanoparticles on intestine inflammation in mice," Journal of Agricultural and Food Chemistry, vol. 67, no. 33, pp. 9382-9389, 2019.

[38] A. Dimitrovska Cvetkovska, S. Manfredini, P. Ziosi et al., "Factors affecting SPF in vitro measurement and correlation with in vivo results," International Journal of Cosmetic Science, vol. 39, no. 3, pp. 310-319, 2017.

[39] B. Gabard and J. Ademola, "Lip sun protection factor of a lipstick sunscreen," Dermatology, vol. 203, no. 3, pp. 244-247, 2001.

[40] D. Voegeli, "The effect of washing and drying practices on skin barrier function," Journal of Wound, Ostomy, and Continence Nursing, vol. 35, no. 1, pp. 84-90, 2008.

[41] J. Blaak and P. Staib, "The relation of $\mathrm{pH}$ and skin cleansing," Current Problems in Dermatology, vol. 54, pp. 132-142, 2018.

[42] A. M. Barbero and H. F. Frasch, "Pig and guinea pig skin as surrogates for human in vitro penetration studies: a quantitative review," Toxicology In Vitro : an international journal published in association with BIBRA, vol. 23, no. 1, pp. 1-13, 2009.

[43] C. Wang, D. Wang, T. Dai et al., "Skin pigmentation-inspired polydopamine sunscreens," Advanced Functional Materials, vol. 28, no. 33, p. 1802127, 2018.

[44] F. Mattiroli, J. H. Vissers, W. J. van Dijk et al., "RNF168 ubiquitinates $\mathrm{K} 13-15$ on $\mathrm{H} 2 \mathrm{~A} / \mathrm{H} 2 \mathrm{AX}$ to drive DNA damage signaling," Cell, vol. 150, no. 6, pp. 1182-1195, 2012.

[45] A. D. Monnot, W. V. Christian, M. M. Abramson, and M. H. Follansbee, "An exposure and health risk assessment of lead $(\mathrm{Pb})$ in lipstick," Food and Chemical Toxicology, vol. 80, pp. 253-260, 2015. 